\title{
INGRESOS DIGITALES - IMPUESTOS ANÁLOGOS
}

\section{DIGITAL INCOME - ANALOG TAXES}

\author{
Javier Mauricio Merino Gonzálezl
}

\section{Resumen}

A mediados de 2019 en Japón, ${ }^{2}$ el G20 acordó crear un impuesto digital (cuyo borrador sería debatido los primeros meses de 2020, e inclusive desde esta fecha ya se pretende empezar a cambiar la legislación en algunos paises), dirigido a las grandes empresas cuyas operaciones se basan principalmente en el internet para generar enormes ingresos como Facebook, Amazon, Google, Netflix, Uber, Spotify, Airbnb, etc. Esta tasa digital está orientada a que: las grandes empresas de la red paguen impuestos no por su ubicación geográfica (residencia fiscal) como se lo hace actualmente, sino con base en el número de usuarios de cada uno de los países en los que brindan el servicio, para evitar de esta forma los vacios legales existentes, por la falta de actualización de las leyes, o la elusión fiscal. Pretendiendo ser más justos al momento de recaudar tributos para todos los actores sin paraisos fiscales, filiales etc. Este trabajo busca contribuir al debate generado en relación a la imposición de esta tasa digital. Este articulo se divide en primera instancia en la revisión de la normativa tributaria del Ecuador, y en segunda instancia la implicación de una tasa digital en las arcas fiscales de nuestro país.

\section{Palabras clave}

Tributos, impuestos, negocios digitales, IVA, IR.

\section{Summary}

In mid-2019 in Japan, the G20 agreed to create a digital tax (the draft of which will be debated in the first months of 2020, and even from this date it is already intended to start changing the legislation in some countries), aimed at large companies our operations rely mainly on the internet, to generate income such as Facebook, Amazon, Google, Netflix, Uber, Spotify, Airbnb, etc. This digital rate is aimed at: large network companies pay taxes not based on their geographical location (tax residence) as it is currently done, but based on the users of each of the countries in which they provide the service, to avoid in this way the existing legal loopholes, due to the lack of updating of the laws. Pretending to be fairer when collecting taxes for all the actors: without tax havens, subsidiaries, etc. This work seeks to contribute to the debate generated in relation to the imposition of this digital rate. This article is divided into the first instance in the review of Ecuador's tax regulations, and in the second instance the implication of a digital rate in the Ecuador's tax coffers.

\section{Keywords}

Taxes, taxes, digital business, VAT, IR.

JEL: $\mathrm{H} 2-\mathrm{H} 25$ 


\section{Introducción}

Dentro de la normativa ecuatoriana todos los sujetos pasivos tenemos que pagar impuesto a la renta, ya sea como personas naturales o jurídicas (Servicio de Rentas Internas, 2017); gracias a estos impuestos el gobierno puede planificar, financiar y ejecutar obras y servicios en beneficio de los ciudadanos (Ministerio de Economía y Finanzas, 2020) para así tener cada vez un mejor nivel de vida.

Si bien, los Estados hacen esfuerzos para garantizar una mejor y mayor calidad de vida (desde el aspecto gubernamental en primera instancia) es, en gran medida, la contribución y el pago correspondiente a los tributos respectivos los que hacen posible o no la consecución de las obras planificadas, y al ser el impuesto a la renta ${ }^{3}$ el $35 \%$ del total de los tributos recaudados en Ecuador en el 2018 (Servicio de Rentas Internas, 2018) el Estado ve la necesidad de ampliar la masa de los contribuyentes, basándose en el primer principio de Generalidad, ${ }^{4}$ como un mecanismo para llegar al último de los principios tributarios y lograr una Suficiencia Recaudatoria ${ }^{5}$ (Servicio de Rentas Internas, 2018).

Tomando en cuenta que una obligación tributaria es el vínculo jurídico entre los sujetos (activo y pasivos) al verificarse el hecho generador (Asamblea Nacional de Ecuador, 2017), de forma imperante, urgente, necesaria, justa (con base en la normativa análoga pero vigente) y equitativa, al producirse el pago por un servicio brindado, o un bien comprado, se derivan consecuentemente la obligación tributaria para las partes involucradas, por un lado los compradores tienen la obligación de cancelar los impuestos que le corres- ponden, como por ejemplo, el impuesto al valor agregado (IVA), y por el otro, los vendedores al ser la actividad objeto del impuesto (Servicio de Rentas Internas, 2017) pese a no ser considerada dentro de la normativa legal (por ser análoga, obsoleta, del siglo pasado) obtienen una renta (sean estos personas naturales o jurídicas, nacionales o extranjeras) y es en este punto en el que nos basaremos para el análisis del presente trabajo.

Por el momento se dejará de lado el tema impositivo (IVA) a los compradores (clientes, usuarios), ya que este se daría como consecuencia natural, simple y sencilla de aplicación, una vez que llegue cada estado (o región) a determinar la actualización al "siglo XXI" de los "tributos digitales" a estas grandes empresas internacionales (o nacionales de ser el caso) (El Universo, 2019), como ya se ha empezado a dar en el Ecuador, gracias a los agentes de retención, como lo indica Juan Carlos Peñafiel (El Universo, 2019).

Según el Servicio de Rentas Internas (SRI) detalló que iniciará el cobro del Impuesto al Valor agregado (IVA) desde septiembre, porque se cumple el plazo establecido de 180 días hábiles, que se dio a los usuarios de plataformas digitales extranjeras por el usos de sus servicios, en concordancia con la Ley Orgánica de Simplificación y Progresividad Tributaria que fue aprobada en diciembre de 2019.

Subirá el gasto para quienes tienen sus cuentas en Netflix, Spotify, Uber, Airbnb, entre otros. Por ejemplo, quienes usan el plan Estándar (dos pantallas para ver al mismo tiempo y en calidad HD) de Netflix, cuyo valor es de USD 10.99, subirá a USD 12.30. En cambio si es un servicio Premium de USD 13.99, pagará USD 15.66. (Comunicaciones, 2020).

USD 5.319 millones de dólares de los Estados Unidos de América.

La Ley Tributaria tiene el carácter de general y abstracta, no está direccionada a una persona o grupo de personas. Principio orientado a que el Estado tenga en cuenta que la recaudación tributaria será suficiente para el financiamiento del presupuesto. 


\section{Cambios precios Netflix en Ecuador}

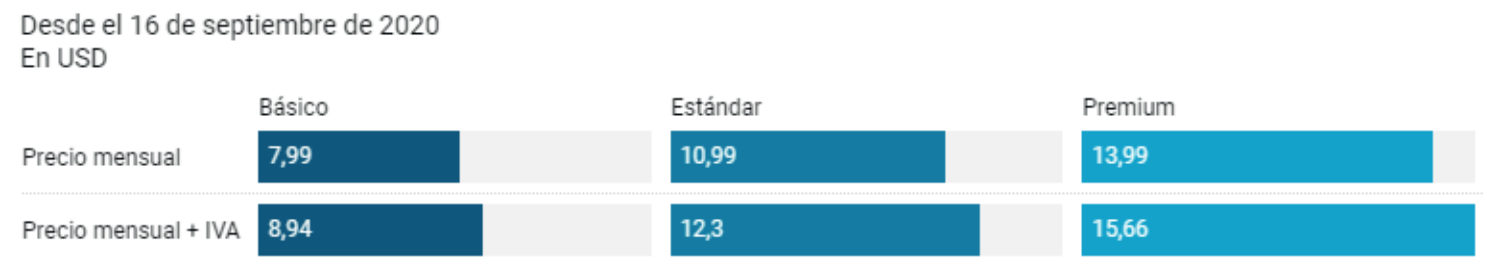

Gráfico: Gabriela Coba - PRIMICIAS · Fuente: Netflix · Descargar los datos · Creado con Datawrapper

Fuente: Netflix

Según el reglamento de la Ley de Simplificación y Progresividad Tributaria, se consideran servicios digitales a "aquellos prestados o contratados a través de Internet, o de cualquier adaptación o aplicación de los protocolos, las plataformas o la tecnología utilizada por Internet u otra red (...) que estén automatizados y requieran una intervención humana mínima". El documento contempla 15 categorías, entre las que se encuentran servicios de software, música, educación, juegos y noticias. (Coba, 2020).

Con esta ley, el gobierno plantea recaudar alrededor del USD 600 millones para el 2020 y así cumplir con los acuerdos pendientes que se tiene con el Fondo Monetario Internacional (FMI) para ese año. "La situación económica del país requiere el aporte de todos para lograr la prosperidad que deseamos. Será un beneficio para los ecuatorianos" (Quito, 2019).

En este punto se centrará esta investigación, ya que si se da el objeto del impuesto (en este caso del impuesto a la renta IR) y por ello, un ingreso (renta) como beneficio por el servicio brindado (así sea en la nube), en última instancia esto quiere decir que efectivamente estas empresas digitales están obteniendo ingresos por su giro del negocio (grandes ingresos) sin pagar los impuestos respectivos en cada uno de los lugares físicos donde se da el hecho generador, y gracias a las artimañas y vacíos de las leyes actuales, se aprovechan para ob- tener mayores utilidades que aquellas empresas establecidas en residencias fiscales (físicas), que si cumplen efectivamente con los pagos claramente estipulados, pagando los impuestos respectivos (financiando al estado para beneficio de todos, incluidos los propios contribuyentes "morosos").

Vale la pena considerar que para los países en vías de desarrollo la composición tributaria con respecto a los impuestos indirectos (IVA) es mayor que aquellos impuestos directos (IR), por este motivo, y con base en el déficit que tienen los países de nuestra región, ampliar la masa así como los bienes y servicios tributables son medidas necesarias para tratar de pasar a superávits (Darío Rossignolo, 2012).

El gran debate internacional de ¿por qué las personas pagan sus impuestos? (Barros Vio, 2013) tiene una gran connotación con el problema del bien común (Loehr y Sandler, 1978), como por ejemplo, los servicios públicos como la luz eléctrica o la defensa nacional, ya que estos no son divisibles, no pueden ser cobrados solo a ciertas personas, no pueden fraccionarse o ser proporcionales para algunos. Al ser público, no se puede impedir el consumo para quienes no pagan, y se ven beneficiados por un servicio gratuito que es asumido por el resto de las personas (naturales o jurídicas).

Tomando en cuenta esto, las personas (naturales o jurídicas) que no contribuyen a las arcas fiscales, obtienen un beneficio de 
forma gratuita exactamente igual a quienes si cumplen con su obligación tributaria respectiva. Más allá de entrar en el debate de si es esto una cuestión legal, moral, ilegal o de inmoralidad; es justamente la falta del pago del impuesto por el hecho generador el que de una u otra forma (compradores y vendedores) no permite obtener los recursos respectivos para una mejor y mayor calidad de vida.

De acuerdo con el dilema del prisionero, ${ }^{6}$ todas las personas estaríamos mejor si pagásemos menos impuestos, sin embargo, si todos pagamos menos, las arcas fiscales de los Estados disminuirían constantemente, hasta el punto en el cual no se tendrían suficientes recursos para realizar las inversiones necesarias, inclusive para la mínima operación de una nación.

Este artículo se divide en dos partes, la primera es una visión general del marco jurídico vigente, abordado no desde el punto de vista legal, sino tributario (impositivo). Y en la segunda parte, se lo analiza desde un punto de vista teórico sobre la aplicación tributaria de un impuesto digital a los grandes ingresos de la industria tecnológica en los países donde operan.

El método utilizado es deductivo, a partir de leyes y principios vigentes en el Estado ecuatoriano principalmente, con recolección de datos bibliográficos tanto primarios como secundarios, tomando en consideración que no hay mucha información oficial de carácter nacional o regional; además de tablas desarrolladas por el autor para ejemplificar el impacto que podrían tener en las arcas fiscales, desarrolladas estadísticamente a partir de la información oficial mundial para ser aplicadas en nuestro contexto.

Se decidió tomar como ejemplo a la empresa Netflix Inc. que es una compañía de entretenimiento estadounidense cuya prin- cipal actividad es la distribución de contenido audiovisual a través de una plataforma en línea (streaming), fundada en agosto de 1997 en California por Reed Hastings y Marc Randolph con ingresos de más de USD 15 mil millones en 2018.

En sus inicios alquilaban DVD por correo, actividad que hasta ahora la mantienen, en el año 2000 introdujeron un sistema de recomendación personalizada. En 2002 Netflix hace su oferta pública inicial en Nasdaq con el símbolo NFLX, a partir de 2007 en Estados Unidos empezaron un servicio de video bajo demanda (VOD), exclusivamente en computadoras, pero a partir de 2008 ampliaron las opciones a televisores, tabletas, consolas de juegos, etc. En 2010 se internacionaliza con su servicio en Canadá. En 2011 por primera vez empezaron sus operaciones fuera de América del Norte, llegando a América Latina y el Caribe, además de empezar con su producción original. En 2012 llegaron a Europa, y desde el 2016 a todo el mundo excepto Crimea, Corea del Norte, China y Siria. En 2017 ganaron su primer Oscar al mejor documental corto, y en 2018 gana un Oscar al mejor documental largo, y se convierte en el servicio con más nominaciones a los premios Emmy.

Actualmente tiene presencia en más de 190 países en todo el mundo.

\section{Ingresos digitales, impuestos análogos}

De acuerdo con el artículo 1 de la Ley de Régimen Tributario Interno del Ecuador: se indica que "el objeto del impuesto a la renta se establece para las sociedades extranjeras de acuerdo con las disposiciones de la presente ley"..., y basándose en el artículo 2 y 8 de la misma ley: se determina que el concepto de renta "son ingresos de fuente ecuatoriana ob-

$6 \quad$ Vale la pena indicar que si bien se podría pensar en que si no pagamos impuestos, igual seguiríamos obteniendo los mismos beneficios por parte del Estado, esto en el corto plazo no podría ser sostenible por ningún gobierno, ya que los fondos son limitados y los gastos e inversiones son constantes, sin tener fuentes de ingresos (tributos: impuesto, tasas, contribuciones, etc.), no es viable continuar con la prestación de servicios por parte del Estado. 
tenidos a título oneroso provenientes del capital y/o del trabajo consistentes en dinero"...

Como se indicó anteriormente este artículo no se basa en el punto de vista legal, pero vale la pena aclarar que este aspecto es fundamental para el análisis del mismo, ya que además de los artículos citados, también hay que hacer referencia al artículo 4.2 de la misma ley, sobre la residencia fiscal para sociedades, puesto que si bien se indica claramente que una sociedad en Ecuador se da cuando la misma se ha constituido o creada en el territorio ecuatoriano con la legislación nacional vigente, es exactamente en este punto donde existen vacíos legales, el cual lo podemos plantear con la siguiente pregunta: ¿para que se constituye una empresa en Ecuador, si toda su operación la pueda hacer (y ya se la hace) desde la nube? Es decir, que no necesita ni requiere una ubicación geográfica para su operación, comercialización, distribución, e inclusive para el soporte y mantenimiento, ya que gracias a la tecnología se ha trascendido a las fronteras físicas, para romper las mismas, así como de las ubicaciones geográficas. Esta pregunta la analizaremos y profundizaremos más adelante.

De la misma forma, el artículo 78 de la Ley de Régimen Tributario Interno indica que el hecho generador de los impuestos, "es el consumo del bien o la prestación del servicio", y es este hecho, el que las empresas digitales no están considerando para el pago respectivo del IR por la obtención de ingresos en la prestación de sus servicios.

Cabe indicar que en la Ley Orgánica de Simplificación y Progresividad Tributaria (Asamble Nacional del Ecuador, 2019), publicada en el Suplemento del Registro Oficial Año I - N. 111 del 31 de diciembre de 2019, se indica:

Artículo 27.- En el artículo 63, efectúense las siguientes reformas: 1. En el literal a.1 inclúyase el siguiente numeral: 3. Los no residentes en el Ecuador que presten servicios digitales conforme se definan en el reglamento a esta ley, siempre y cuando se registren en la forma establecida por el Servicio de Rentas Interna.

Lo que indica que aquellas plataformas que así lo hagan como Cabify o Rappi, se sujetaran a las medidas ya estipuladas y establecidas de manera "tradicional", por lo que este trabajo pretende abordar para todas aquellas que aún no lo han hecho, o por el contrario, busca una nueva normativa legal, diferente, $y$ que se ajuste a los negocios en línea.

Los impuestos "análogos" se ven limitados por no poder evolucionar con la velocidad con la que las empresas lo hacen para generar cada vez más negocios de forma globalizada, ya que en cuestión de minutos se pueden generar "ventas" internacionales que cruzan literalmente todo el planeta, para generar una transacción, a través del internet, compra-venta de bienes o servicios, es decir vemos como se da un hecho generador sin presencia física de las partes.

\section{Aplicaciones}

A partir de este punto retomaremos el tema impositivo, que sin lugar a duda, es necesario aclarar que con las leyes actuales las personas jurídicas, al tener actividades lícitas en Ecuador, obtienen ingresos a cambio de bienes o servicios, y por este hecho (generador) tienen y deben contribuir a través de los tributos respectivos; exactamente de igual manera como lo hacemos las personas naturales, al obtener ingresos debemos y tenemos que pagar tributos, todos con el fin de contribuir a la misma sociedad en la que nos desenvolvemos. No es viable que por vacíos legales haya empresas que no paguen impuestos y que paguen menos impuestos inclusive que las mismas personas naturales, es decir que obtienen grandes ingresos, pero por artimañas legales, monetariamente contribuyen con un valor muy inferior al que les corresponde. 
Cabe recalcar lo indicado por el diario económico español Cinco Días (Ricardo Sobrino, 2019) con respecto a los tributos pagados por Netflix España en el 2018, cuyo valor fue de apenas 3146 euros, el equivalente a lo que un trabajador español que gana 24000 euros al año cancela en su declaración de impuesto.

Esto gracias a que desde que ingresó al mercado español, su facturación fue realizada a través de una sociedad Holandesa (Netflix International B.V.), con lo que el beneficio generado por el negocio en el mercado local (España) escapaba a la tributación española.

Netflix creó dos empresas en España el año anterior, una dedicada a la producción y la otra al marketing, las cuales desde su creación en el mes de agosto hasta diciembre de 2018 (ambas filiales), solo declararon ingresos por un poco más de medio millón de euros, y beneficios netos de menos de 10000 euros, cifras que sin lugar a duda contrastan drásticamente con el poderío de la multinacional. Siguiendo un esquema similar al de otras grandes empresas como Google, Amazon, Facebook o Apple, sirviéndose de "estructuras" para pagar menos impuestos (Rasteletti, Pineda y Seira, 2019).

En nuestro caso ecuatoriano, para poder contextualizar y entender la magnitud de las cifras que estas grandes empresas manejan, realizaremos una pequeña comparación de forma muy generalizada.

Adjunto se detalla la Recaudación Acumulada en el 2018 de acuerdo con la información oficial del SRI proporcionada por la Dirección de Planificación y Gestión Estratégica, con respecto a todos los tributos ${ }^{7}$ recaudados a nivel nacional:

Tabla 1. Recaudación tributaria en el Ecuador en el año 2018

\begin{tabular}{|l|r|}
\hline \multicolumn{1}{|c|}{ Provincia } & \multicolumn{1}{|c|}{ USD } \\
\hline Azuay & 754.691 .846 \\
\hline Bolívar & 11.927 .593 \\
\hline Cañar & 35.565 .834 \\
\hline Carchi & 20.202 .454 \\
\hline Chimborazo & 81.819 .070 \\
\hline Cotopaxi & 128.205 .953 \\
\hline El Oro & 216.714 .703 \\
\hline Esmeraldas & 61.090 .693 \\
\hline Galápagos & 33.898 .751 \\
\hline Guayas & 4.388 .265 .999 \\
\hline hnbabura & 109.270 .147 \\
\hline Loja & 74.921 .042 \\
\hline Los Ríos & 79.469 .734 \\
\hline Manabí & 284.484 .453 \\
\hline Morona Santiago & 15.939 .107 \\
\hline Napo & 11.048 .512 \\
\hline Orellana & 26.707 .035 \\
\hline Pastaza & 13.034 .492 \\
\hline Pichincha & 8.313 .039 .339 \\
\hline Santa Elena & 33.865 .978 \\
\hline Santo Domingo De Los Tsáchilas & 83.535 .128 \\
\hline Sin asignar & 1.595 .532 \\
\hline Sucumbíos & 21.567 .267 \\
\hline Tungurahua & 240.853 .938 \\
\hline Zamora Chinchipe & 103.354 .517 \\
\hline Total Recaudación & 15.145 .069 .116 \\
\hline
\end{tabular}

Fuente: Elaborado por el autor

$7 \quad$ Tributos: no solo se considera el Impuesto a la Renta sino todos los tributos. 
Las provincias de Napo y Bolívar que están resaltadas en el cuadro, van a servir como un ejemplo para tomar en consideración con respecto al estudio del presente trabajo.

Para realizar el estudio de dicho análisis se ha elaborado un cuadro referencial para estudiar el impacto al que se hace referencia en la investigación, tomando como ejemplo solo a una de estas grandes empresas internacionales (Netflix, 2019):

Tabla 2. Posible recaudación trinutaria en el Ecuador ${ }^{8}$

\begin{tabular}{|l|r|r|}
\hline & Mundo & Ecuador \\
\hline Habitantes & 7700000000 & 17000000 \\
\hline Clientes & 167000000 & 368701 \\
\hline Porcentaje & $2.2 \%$ & $2.2 \%$ \\
\hline Pago promedio USD & 10.99 & 10.99 \\
\hline Ingreso mensual USD & 1835330000 & 4052027 \\
\hline Meses & 12 & 12 \\
\hline Ingreso anual USD & 22023960000 & 28624327 \\
\hline \% Impuesto Renta & & 12156082 \\
\hline Impuesto USD & & \\
\hline
\end{tabular}

Fuente: Elaborado por el autor

De los 7700 millones de personas que hay en el mundo (Wikipedia, 2019), un $2.2 \%$ tienen una suscripción a Netflix hasta el año 2019, es decir 167 millones de clientes en todo el mundo (El Comercio, 2020). A un precio promedio de USD 10.99 de los servicios que oferta la plataforma, mensualmente obtienen ingresos de mil ochocientos millones (dólares de los Estados Unidos de América), lo que al año se transforma en la increíble suma de más de 22000 millones de dólares (más de lo recaudado en todo Ecuador por cuestión de todos los tributos).

Lamentablemente no se dispone de información oficial en Ecuador de la cantidad de suscriptores que tiene Netflix, por lo que utilizando la misma estadística (solo de manera referencial) para hacer un análisis del impacto de los tributos en las plataformas digitales, en cada uno de los países donde operan (y no pagan ningún tipo de impuesto a la fecha en la mayoría de nacio- nes), podemos deducir que en Ecuador hay más de 360000 suscriptores (2.2\% de la población) (Wikipedia, 2019). Lo cual mensualmente da un ingreso de más de 4 millones de dólares, que al año serían más de 48 millones de dólares.

Como indicamos anteriormente, al no contar con información oficial, y solo de manera informativa para poder contextualizar las grandes cantidades de dinero que se mueven en la nube gracias al internet y la globalización en general, el $25 \%$ del IR que pagan en Ecuador las empresas "físicas", este representaría la cantidad de más de 12 millones de dólares al año; si bien es cierto este valor se lo está asumiendo solo sobre los ingresos totales (estimados) sin considerar los costos y gastos para llegar a la base imponible del impuesto para el cálculo respectivo, cabe profundizar un poco más en este punto sobre la pregunta planteada anteriormente: ¿Qué costos y gastos

8 Cuadro desarrollado de manera referencial para contextualizar los posibles tributos en Ecuador. 
podrían tener estas empresas en cada uno de los países donde operan?, y esta pregunta nos lleva a dos consideraciones a tomar en cuenta, la cual se deriva una de la otra:

a. Hoy en día, estas empresas digitales, no tienen absolutamente ninguno de estos conceptos (valores) y brindan sus servicios sin problema, ya que justamente gracias a la tecnología de sus plataformas no es necesario contar con presencia física.

b. Si no es necesario un costo, un gasto físico en cada país, el cálculo realizado en el cuadro anterior de manera sencilla, referencial y contextualizada para nuestra investigación, parecería tener un peso más relevante y un valor un poco más aproximado y real de lo que podrían obtener las arcas fiscales de las naciones con el aporte tributario de estos nuevos impuestos digitales.

Adicionalmente, vale la pena tomar en consideración que todos los costos y gastos, que podrían tener las empresas para sus operaciones nacionales, como lo determinan las leyes actuales de manera justa, al igual que todas las demás empresas, habría que considerar una vez más que no se manejen al filo de la norma para transferir, prestar o hacer cualquier otra artimaña contable (Seira, 2019) para, por ejemplo, disminuir el valor de la base imponible y poder llegar a lo mismo, es decir pagar valores muy pequeños de tributos (como en España), o inclusive no pagar absolutamente nada como actualmente ocurre.

Si bien es cierto, para que todo esto suceda, hay que cambiar, actualizar y mejorar las normativas tributarias, como hemos visto anteriormente, es decir desde un punto de vista legal, esta investigación no se enfoca en este aspecto, sino en el tributario. Es en este punto en donde de manera comparativa y referencial tiene importancia el primer cuadro de los Ingresos Tributarios obtenidos en Ecuador en el 2018; las provincias de Napo y Bolívar obtuvieron ingresos por cuestión de todos los tributos recaudados (no solo por el IR) por valores en el primer caso inferiores a los que podría aportar esta gran empresa digital y apenas un poco más en comparación con la provincia de Bolívar, solo tomando como referencia inicial a una de estas compañías, el cual se debería aplicar para todos, por lo que el valor tranquilamente podría ser exponencial.

$Y$ es justamente en esta pequeña, simple y breve comparación, entre los ingresos obtenidos por dos provincias enteras del Ecuador, y lo que podrían ser los tributos sobre los ingresos de los negocios digitales, donde sin lugar a dudas se puede observar la importancia de tomar cartas en el asunto, para de manera justa, equitativa y progresiva actualizar las leyes, reglamentos y códigos a una era digital del siglo XXI, acorde con la gran transformación y generación de negocios de las empresas en la web.

\section{Reflexiones finales}

Esta investigación pretende buscar un punto intermedio entre todos los actores involucrados en el ámbito tributario de las naciones y en su economía, ya que uno de los primeros temas a considerar es que las empresas digitales al tener grandes recursos, ser multinacionales y contar con personas muy preparadas y, sobre todo, actualizadas tienen sin lugar a duda, una gran ventaja frente a prácticamente todas las leyes de cualquier nación, ya que por más desarrollado que sea un país en que pueda ejercer, su capacidad operativa, legal y su propia burocracia no facilitan las cosas para ir al mismo ritmo y tecnología que estas grandes empresas multinacionales.

Así como las empresas de la nube han evolucionado de manera exponencial, aunque 
un poco más tarde, las naciones, y que mejor que las regiones en conjunto puedan dar el gran salto para actualizar sus leyes y reglamentos a la misma velocidad y con el mismo grado de amplitud y efectividad, para de forma progresiva, generalizada y equitativa hacer que los ingresos digitales estén acorde con los impuestos de los nuevos retos y de los nuevos negocios o formas de negocio.

Si bien es cierto no es una tarea fácil, por la cantidad de involucrados y la misma complejidad de obtener nuevas normas, leyes, reglamentos y reglas de juego claras y precisas para todos, la única forma de llegar a un gran acuerdo internacional es buscar consensos entre las partes para tomar en cuenta a todos los actores económicos (Economía, 2019).

Valdría la pena preguntarnos, si es necesario aterrizar a las empresas de la nube a la tributación actual, o si por el contrario necesitaríamos que sean los tributos los que suban a la nube.

\section{Propuesta}

Con el presente trabajo, se pretende en última instancia aportar con una propuesta que busque relacionar a las partes involucradas de una manera justa, sin perjudicar a nadie, y esto se lo podría hacer con las siguientes consideraciones, una vez más desde el punto de vista tributario (más que desde el punto de vista técnico):

Incorporar al artículo 4.2 de la Ley de Régimen Tributario Interno del Ecuador que se pueda considerar a "Empresas Digitales" que sin haber sido constituidas físicamente en Ecuador, puedan registrarse a través de un "RUC Digital" del SRI y con la ayuda, supervisión y control de la ARCOTEL ${ }^{9}$ para que sean consideradas dentro de la nueva normativa tributaria.
Con este registro digital se pretende que todas aquellas empresas extranjeras que no tengan un RUC (tradicional) debidamente registrado para su operación diaria puedan funcionar legalmente en Ecuador, y a través de este registro digital cargar con un Impuesto Digital sobre las ventas realizadas en el territorio.

Todo esto solo se lo puede conseguir con la ayuda y colaboración de todos los entes reguladores del Estado, ya que la ARCOTEL debería normalizar a través de los proveedores de internet para que, aquellas empresas de la nube que no se han registrado digitalmente, sean bloqueadas sus IP hasta que realicen los registros respectivos (físicos o digitales) (Zavia, 2016).

Con esto no se pretende coartar las libertades ni de las empresas, ni de los clientes, lo que se busca es tomar medidas suficientes, necesarias, diferentes, que no son fáciles de aplicar, para integrar y garantizar una libre competencia entre todos los actores socio económicos.

Según diario El Universo, el viceministro de Finanzas dijo que "el concepto en general es que todas las actividades sean formalizadas, a fin de igualar las condiciones en el mercado" (El Universo, 2019).

Al ser consultado sobre el tema del incremento del IVA, acerca de eliminar exenciones del IR, que son temas que también se podrían incluir en la reforma, Carrillo ratificó que este tema está en análisis. Explicó, también, que lo que se busca es un consenso entre diversos actores, especialmente en la Asamblea, por lo que prefiere no adelantar este tipo de temas (El Universo, 2019)

\section{Referencias}

Asamblea Nacional del Ecuador. (2019). Ley Orgánica de Simplificación y Progresividad Tributaria. Suplemento del Registro Oficial.

$9 \quad$ Agencia de Regulación y Control de las Telecomunicaciones. 
31 de diciembre. Obtenido de https://www. sri.gob.ec/web/guest/ley-organica-desimplificacion-y-progresividad-tributaria

Asamblea Nacional de Ecuador. (2017). Ley de Regimen Tributario Interno. Abril. Obtenido de file://C:/Users/avernaza/Downloads/ LEY\%20DE\%20R\%C3\%89GIMEN\%20TRIBUTARIO\%20INTERNO_abril\%202017.pdf

Barros Vio, B. (2013). ¿Porque las personas pagan sus impuestos? Subjetividad y Procesos Cognitivos 17(2), 31-47.

Coba, G. (2020). Lista de servicios digitales que pagan IVA se actualizará cada tres meses. Primicias Ec. 19 de agosto. Obtenido de https://www.primicias.ec/noticias/economia/sri-lista-servicios-digitales-iva/

Comunicaciones, P. (27 de 06 de 2020). Pichincha Comunicaciones. Obtenido de http://www. pichinchacomunicaciones.com.ec/el-sridesde-septiembre-cobrara-iva-a-usuariosde-plataformas-digitales-extranjeras/

Economía. (2019). Ya hay fecha para ordenar que Claro, Tigo y Movistar bloqueen Uber de los celulares. Head Topics. https://headtopics.com/co/ya-hay-fecha-para-ordenarque-claro-tigo-y-movistar-bloqueen-uberde-los-celulares-pulzo-com-10320857

El Comercio. (2020). Netflix alcanza $167 \mathrm{mi}-$ llones e suscripciones, pero vislumbra crecimiento más lento. El Comercio. 22 de enero. Obtenido de https://www.elcomercio.com/tendencias/netflix-aumentosuscriptores-crecimiento-competencia. html\#: : :text=Netflix\%20alcanza\%20 $167 \% 20$ millones\%20de\%20suscripcio nes\%2C\%20pero\%20vislumbra\%20crecimiento\%20m\%C3\%A1s\%20lento,-777 \&text=Netflix\%20inform\%C3\%B3\%20 este $\%$

El Universo. (2019). ¿Qué servicios digitales serán gravados con el 12\% del IVA? El Universo. 23 de diciembre. Obtenido de https://www. eluniverso.com/noticias/2019/12/19/ nota/7658049/servicios-digitales-iva-tributaria-reforma-ecuador-uber-netflix

El Universo. (2019). El Universo: Servicios Digitales 12\% IVA. El Universo. 23 de diciembre. Obtenido de https://www.eluniverso.com/ noticias/2019/12/19/nota/7658049/ servicios-digitales-iva-tributaria-reformaecuador-uber-netflix

El Universo. (2019). ¿Pagaría usted impuestos por Netflix, Spotify, Cabify o Uber? El Universo. 27 de junio. Obtenido de https://www. eluniverso.com/noticias/2019/06/27/ nota/7398240/gobierno-analiza-impuestos-servicios-digitales-dice-viceministro

Loehr, W. y Sandler, T. (1979). Public Goods and Public Policy. The American Political Science Review. 73(4), 1188-1189. DOI: HYPERLINK "https://doi.org/10.2307/1954055" \t "_blank" https://doi.org/10.2307/1954055

Ministerio de Economía y Finanzas. (2020). Presupuesto General del Estado 2020. Obtenido de https://www.finanzas.gob.ec/wpcontent/uploads/downloads/2020/01/ cifras-pge-2020_compressed-1-10.pdf

Radio La Calle. (2019). Ley tributaria pone impuesto a las plataformas digitales. Radio La Calle Quito. 11 de diciembre. Obtenido de https://radiolacalle.com/ley-tributaria-pone-impuesto-a-las-plataformas-digitales/

Rasteletti, A.; Pineda, E. y Seira, E. (2019). CIAT. Obtenido de Centro Interamericano de Administraciones Tributarias. https://www. ciat.org/retos-para-el-cobro-del-impuesto-sobre-la-renta-en-la-economia-digital/

Ricardo Sobrino. (2019). Netflix tan solo paga 3.146 euros de impuestos en España en su primer ejercicio fiscal. Cinco Diaz. 28 de junio. Obtenido de Cinco Diaz: https://cincodias.elpais.com/cincodias/2019/06/27/ companias/1561661679_765532.html

Rossignolo, D. (2012). Estimación de la recaudación potencial del impuesto a la renta en América Latina. CEPAL 20.

Seira, E. P.-A.-E. (2019). Recaudando Bienestar. 15 de noviembre. Obtenido de https://blogs. iadb.org/gestion-fiscal/es/impuesto-sobre-la-renta-en-la-economia-digital/

Servicio de Rentas Internas. (2017). Ley Orgánica de Regimen Tributario Interno. Registro Oficial Suplemento 463. 29 de diciembre.

Servicio de Rentas Internas. (2018). Direccion Nacional de Planificacion y Gestion Estrategica. Estadisticas de Recaudacion. 31 de diciembre. 
Servicio de Rentas Internas. (2018). Codigo Tributario. 21 de agosto. Obtenido de https:// www.ces.gob.ec/lotaip/2018/Agosto/ Anexos-literal-a2/CODIGO\%20TRIBUTARIO.pdf

Wikipedia. (Diciembre de 2019). La enciclopedia libre. Obtenido de https://es.wikipedia. org/wiki/Poblaci\%C3\%B3n_mundial\#cite_ note-Poblaci\%C3\%B3n_mundial-3
Wikipedia. (2019). La enciclopedia libre. Obtenido de https://es.wikipedia.org/wiki/ Demograf\%C3\%ADa_del_Ecuador

Zavia, M. S. (2016). Corea del Norte, un país sin Internet, bloquea el accso a Facebook, YouTube y Twitter. Gizmodo. 4 de enero. Obtenido de https://es.gizmodo.com/ corea-del-norte-un-pais-sin-internet-bloquea-el-acces-1768424028 